\title{
O peso da mente feminina: associação entre obesidade e depressão
}

Sónia Martins*

\section{RESUMO}

Objectivos: Determinar a prevalência de obesidade numa USF. Determinar a prevalência de depressão em obesos numa USF. Verificar se existe associação entre obesidade e depressão e se esta é diferente entre géneros.

Tipo de estudo: Observacional analítico transversal

Local: USF BRIOSA, Coimbra

População: Utentes inscritos na USF em Novembro de 2010, com idade superior a 18 anos e com registo válido do índice de massa corporal (IMC) nos últimos 12 meses. Excluíram-se todas as mulheres que estiveram grávidas nalgum período, durante os últimos 12 meses.

Métodos: Os dados foram recolhidos do programa informático MedicineOne ${ }^{\circledR}$. Variáveis: género, IMC e depressão. A análise estatística incluiu o cálculo do Odds ratio.

Resultados: A percentagem de mulheres na população foi de $62,18 \%$. A prevalência de obesidade na população foi $18,67 \%$. A prevalência de depressão em obesos foi 30,82\%. Verificou-se uma associação entre obesidade e depressão na população estudada $(O R=1,33)$. Quando se fez o estudo por géneros, apenas no grupo das mulheres se verificou esta associação $(O R=1,51)$. Conclusões: A prevalência da obesidade na USF foi superior à encontrada noutros estudos nacionais. Não foram encontrados estudos para comparação da prevalência de depressão em obesos. Os resultados encontrados poderão contribuir para a adopção de estratégias preventivas e tratamentos mais eficazes, diferentes entre géneros.

Palavras-chave: Obesidade; Depressão.

\section{INTRODUÇÃO}

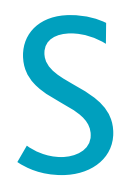
egundo a Organização Mundial da Saúde (OMS), a obesidade é definida como uma doença em que o excesso de gordura corporal acumulada pode atingir graus capazes de afectar a saúde. O excesso de gordura resulta de sucessivos balanços energéticos positivos, em que a quantidade de energia ingerida é superior à quantidade de energia dispendida. Os factores que determinam este desequilíbrio são complexos e incluem factores genéticos, psicológicos, socioeconómicos, culturais e ambientais. Este desequilíbrio tende a perpetuar-se, pelo que a obesidade é considerada uma doença crónica.

O diagnóstico da obesidade é realizado a partir do índice de massa corporal (IMC), que se calcula pela fór-

* Médica Interna de Medicina Geral e Familiar

Unidade de Saúde Familiar BRIOSA, ACES Baixo Mondego I mula $\left[\mathrm{IMC}=\right.$ Peso $(\mathrm{kg}) /$ Altura $\left.\left(\mathrm{m}^{2}\right)\right]$. Através deste parâmetro, são considerados obesos os indivíduos cujo IMC se encontra num valor igual ou superior a 30 $\mathrm{kg} / \mathrm{m}^{2}$. Podem-se igualmente definir vários níveis de obesidade de acordo com o IMC: grau 1 (entre 30 e 34,9 $\mathrm{kg} / \mathrm{m}^{2}$ ); grau 2 (entre 35 e $39,9 \mathrm{~kg} / \mathrm{m}^{2}$ ); grau 3 (superior a $\left.40 \mathrm{~kg} / \mathrm{m}^{2}\right)$.

Nas últimas décadas a obesidade tem adquirido proporções epidémicas e a OMS reconhece que se não forem tomadas medidas drásticas para prevenir e tratar a obesidade, mais de $50 \%$ da população mundial será obesa em 2025. Em 2008, 10\% da população masculina e $14 \%$ da população feminina, ${ }^{1}$ a nível mundial, era obesa, segundo esta organização.

Segundo o boletim da Sociedade Portuguesa Para o Estudo da Obesidade (SPEO), ${ }^{2}$ no ano de 2000, a prevalência da obesidade era de $12,9 \%$ nos homens e $15,4 \%$ nas mulheres. No Inquérito Nacional de Saúde 
$2005 / 2006,{ }^{3}$ estimava-se que $15,2 \%$ dos residentes em Portugal, com mais de 18 anos, eram obesos.

Em 2008, foi publicado um estudo de Isabel do Carmo e colaboradores, ${ }^{4}$ que considera que entre, $2003 \mathrm{e}$ $2005,14,2 \%$ da população portuguesa era obesa.

Esta patologia leva a um aumento da prevalência da diabetes, hipertensão arterial, dislipidémia, hiperuricemia, litíase vesicular, síndrome do ovário poliquístico, doença coronária, doença vascular cerebral e alguns tipos de cancro. ${ }^{5}$ Está ainda associada a dificuldades respiratórias e do aparelho locomotor e a um aumento significativo da mortalidade.

A nível psicológico, a alteração da imagem corporal resultante do aumento de peso poderá provocar uma desvalorização da auto-imagem e do auto-conceito, no obeso, diminuindo a sua auto-estima. Em consequência, poderão surgir sintomas depressivos e ansiosos, uma diminuição da sensação de bem-estar e um aumento da sensação de inadequação social, com uma consequente degradação da relação interpessoal.

Enquanto as consequências físicas da obesidade são bem estabelecidas, a relação entre a obesidade e a depressão ainda é incerta e os resultados relatados não têm sido consensuais.

Em 2008, Atlantis E. e colaboradores ${ }^{6}$ concluíram $^{2}$ que, em geral, havia um fraco nível de evidência que apoia a hipótese da associação entre obesidade e depressão e que seriam necessários mais estudos para estabelecer essa relação de causalidade.

Recentemente, Luppino e colaboradores ${ }^{7}$ realizaram uma meta-análise que veio confirmar a associação entre depressão e obesidade e a reciprocidade desta relação.

Sendo a obesidade uma patologia muito frequente, associada a elevada morbilidade e mortalidade, surgiu a hipótese de estudar a associação entre a obesidade e a depressão, para melhor adequação das estratégias preventivas e terapêuticas, caso esta associação se verificasse. Assim, com este trabalho pretendeu-se:

- determinar a prevalência de obesidade e de depressão em obesos na USF BRIOSA;

- verificar se existe associação entre obesidade e depressão e se esta é diferente entre géneros.

\section{MÉTODOS}

Foi realizado um estudo analítico, observacional e transversal na USF BRIOSA durante o mês de Novem- bro de 2010.

A área de influência da USF BRIOSA abrange essencialmente freguesias da cidade de Coimbra. Aproximadamente um terço da população inscrita tem o ensino médio ou superior sendo que quase metade da população trabalha ao nível do sector terciário. A taxa de desemprego, ainda que crescente nos últimos anos, é inferior à nacional.

A população do estudo correspondeu aos utentes inscritos na USF BRIOSA, em Novembro de 2010, com idade superior a 18 anos e com, pelo menos, um registo do IMC nos últimos 12 meses (01 de Novembro de 2009 a 30 de Novembro de 2010). No caso de haver mais que um registo, considerava-se o mais recente. Foram excluídas todas as mulheres que estiveram grávidas nalgum período, durante os últimos 12 meses. Toda a população foi estudada, perfazendo um total de 5700 utentes.

Foram estudadas as seguintes variáveis: género (feminino e masculino), IMC e depressão. A variável depressão foi definida como a codificação de P76 na lista de problemas activos, segundo a 2. ${ }^{a}$ edição da Classificação Internacional de Cuidados Primários (ICPC-2).

Os dados foram recolhidos através do programa informático MedicineOne ${ }^{\circledR}$ e tratados no programa estatístico Epi info®. A análise estatística incluiu o cálculo do Odds Ratio (OR) bruto.

\section{RESULTADOS}

Foram estudados 5700 utentes, dos quais $62,2 \%$ eram do género feminino. A idade variou entre os 18 e os 98 anos, com idade média de 47,8 anos. A prevalência de obesidade na população foi de $18,7 \%$. A prevalência de depressão nos obesos da população estudada foi 30,8\%.

Foi feita análise estatística para verificar se existia alguma associação entre obesidade e depressão na população estudada (Quadro I) através do cálculo do Odds Ratio $(\mathrm{OR}=1,33)$.

Quando se fez a estratificação por géneros (Quadro II) verificou-se que a associação entre obesidade e depressão apenas se verificava no género feminino $(\mathrm{OR}=$ 1,51 versus $\mathrm{OR}=1$ ).

\section{DISCUSSÃO}

Neste estudo foi encontrada uma prevalência da obesidade de $18,7 \%$, superior à encontrada noutros es- 
QUADRO I. Percentagem de doentes codificados com P76 por IMC.

\begin{tabular}{l|c|c} 
& $\mathbf{n}$ & (\%) P76 \\
\hline $\mathrm{IMC}>30$ & 1064 & 30,8 \\
\hline $\mathrm{IMC}<30$ & 4636 & 25,1 \\
\hline & & OR $=1,33$ \\
\hline
\end{tabular}

QUADRO II. Percentagem de doentes codificados com P76 por IMC e género.

\begin{tabular}{l|c|c} 
& $\mathbf{n}$ & $\mathbf{( \% )} \mathbf{P 7 6}$ \\
\hline Sexo Feminino & & \\
\hline $\mathrm{IMC}>30$ & 653 & 41,8 \\
\hline $\mathrm{IMC}<30$ & 2891 & 32,2 \\
\hline Sexo Masculino & & OR $=1,51$ \\
\hline $\mathrm{IMC}>30$ & 412 & 13,4 \\
\hline $\mathrm{IMC}<30$ & 1744 & 13,4 \\
\hline & & OR $=1$ \\
\hline
\end{tabular}

tudos nacionais, nomeadamente no Inquérito Nacional de Saúde 2005/2006, que foi de 15,2\%. Isabel do Carmo e colaboradores consideraram que, entre 2003 e 2005, 14,2\% da população portuguesa era obesa. Com base no Observatório de Saúde Global da OMS, os valores encontrados também eram superiores, visto que, em 2008 , consideraram que $10 \%$ dos homens e $14 \%$ das mulheres, a nível mundial, eram obesos. Os intervalos de confiança não foram incluídos uma vez que toda a população foi estudada.

Consideram-se como aspectos positivos deste estudo o facto de ser um estudo inovador em Portugal, da dimensão da população estudada e de não haver erro amostral.

Este estudo poderá ter algumas limitações. Apenas participaram no estudo utentes com registo de IMC, o que pode, de alguma forma, sobrestimar a prevalência. É o caso de adultos jovens sem patologias em que o exame periódico de saúde recomendado não é anual. Se este subgrupo for excluído, as prevalências de obesidade e depressão serão diferentes, o que altera as es- timativas das variáveis.

A presença de um código ICPC-2 pode não reflectir a prevalência exacta da depressão na população estudada. O código P76 pode permanecer na lista de problemas do doente após remissão clínica da doença. Por outro lado, poderão existir pessoas com diagnóstico de depressão que não tenham o código P76 registado. Nesse caso seria útil incluir as pessoas com prescrição crónica de antidepressivos sem codificação do ICPC-2 associada. Embora tivesse que se ter em conta o uso de antideressivos para patologias que não a depressão, tais como fibromialgia, profilaxia da enxaqueca, bulimia, por exemplo. Também seria útil incluir as pessoas com P03 (Sensação de depressão).

Segundo a ICPC-2, a codificação de P76 inclui também depressão pós-natal/puerperal e neste estudo não foi tido em conta este aspecto. No puerpério muitas mulheres ainda não voltaram ao peso habitual. No entanto, uma vez que foram excluídas as mulheres que estiveram grávidas durante o período do estudo, o impacto deste factor é pequeno.

A colheita de dados foi feita através dos registos da plataforma informática, podendo-se, dessa forma, estar perante um viés de informação. Poderão existir duas variáveis de confundimento, uma vez que a presença de outras comorbilidades não foi tida em conta, assim como o tipo de antidepressivos. Sabe-se que algumas classes de antidepressivos podem levar a aumento de peso e algumas comorbilidades podem limitar a mobilidade e desta forma influir no peso. Teria sido mais vantajoso usar o OR ajustado por modelo de regressão logística.

Os resultados encontrados poderão contribuir para a adopção de estratégias preventivas e tratamentos mais eficazes que poderão ser diferentes entre géneros, com melhores resultados em saúde.

\section{REFERÊNCIAS BIBLIOGRÁFICAS}

1. World Health Organization. Global Health Observatory (GHO). Obesity. Disponível em: http://www.who.int/gho/ncd/risk_factors/obesity_text/en/ [acedido em 30/08/2011].

2. Carmo I, Carreira M, de Almeida MD, Lima Reis JP, Medina JL, Galvão Teles A. Estudo da prevalência da obesidade em Portugal. Bol SPEO 2000: 3-5.

3. Nacional de Saúde Dr. Ricardo Jorge. Inquérito Nacional de Saúde 2005/2006. Lisboa, INSA; 2009. Disponível em: http://www.insa.pt/ sites/INSA/Portugues/Publicacoes/Outros/Paginas/INS2005- 
2006.aspx [acedido em 30/08/2011].

4. Carmo I, Santos O, Camolas J, Vieira J, Carreira M, Medina L, et al. Overweight and obesity in Portugal: national prevalence in 2003-2005. Obes Rev 2008 Jan; 9 (1): 11-9.

5. Direcção-Geral da Saúde. Divisão de Doenças Genéticas, Crónicas e Geriátricas. Programa nacional de combate à obesidade. Lisboa: DGS; 2005. Disponível em: http://www.dgs.pt/upload/membro.id/ficheiros/ i008253.pdf [acedido a 30/08/2011].

6. Atlantis E, Baker M. Obesity effects on depression: systematic review of epidemiological studies. Int J Obes (Lond). 2008 Jun; 32 (6): 881-91.

7. Luppino FS, de WIT Im, Bouvy PF, Stijnen T, Cuijpers P, Penninx BW, et al. Overweight, obesity, and depression: a systematic review and metaanalysis of longitudinal studies. Arch Gen Psychiatry 2010 Mar; 67 (3): 220-9.

\section{CONFLITOS DE INTERESSE}

A autora declara não possuir qualquer tipo de conflitos de interesse.

\section{ENDEREÇO PARA CORRESPONDÊNCIA}

Sónia Martins

Unidade de Saúde Familiar BRIOSA

Avenida Mendes Silva

3030-393 Coimbra

E-mail: soniamartinsusf@gmail.com

Recebido em 02/09/2011

Aceite para publicação em 11/05/2012

\begin{abstract}
Objectives: To determine the prevalence of obesity in a Family Health Unit, to determine the prevalence of depression in obese patients, and to test the association between obesity and depression by gender.

Type of study: Transversal analytical study

Setting: USF BRIOSA, Coimbra, Portugal

Population: Patients over 18 years old registered in USF BRIOSA with their body mass index (BMI) recorded during last year. Methods: Data were gathered from clinical records using the MedicineOne ${ }^{\circledR}$ electronic medical record system. The variables studied included gender, BMI and depression. Statistical analysis included calculation of the odds ratio.

Results: The prevalence of obesity in the population was $18.67 \%$. The prevalence of depression in obese patients was $30.82 \%$. An association between obesity and depression was found in this population $(O R=1.33)$. A significant association between obesity and depression was found only for females $(O R=1.51)$.

Conclusions: The prevalence of obesity in this population was higher than that found in other studies. No studies were found comparing the prevalence of depression in obese and non-obese patients. The results of this study may contribute to the adoption of preventive strategies and increase effectiveness of the treatment by gender.
\end{abstract}

Keywords: Obesity; Depression. 